\section{PTH-005 VERIFYING PATIENT REPORTED FAMILY HISTORY IN THE COLORECTAL FAMILY HISTORY SCREENING CLINIC (CFHSC)}

doi:10.1136/gutjnl-2013-304907.493

1,"M Pettman, 'J H Scholefield. 'Colorectal, Nottingham University Hospitals, Nottingham, UK

Introduction National Colorectal Family History Screening guidelines categorise risk based on incidence of familial cancer, type of cancer, age and genetic proximity. High and Medium risk patients are recommended regular or one off screening colonoscopies. Appropriate assessment of risk involves obtaining detailed, relevant and accurate family history information. Incorrect assessment of risk can result in High/Medium risk patients not receiving tests or Low risk patients being subjected to unnecessary invasive and costly colonoscopies. The majority of asymptomatic referrals to the Nurse led CFHSC are from primary care. The family history information supporting referral provided by GP's is non-standardised. Data provided can often be inaccurate for such reasons as; patients quote the wrong types of cancer, do not relate non bowel associated cancers, mistaking other benign bowel disorders for malignant disorders, and include distant family or family beyond age thresholds. The CFHSC expends significant resource in improving the accuracy and relevance, with respect to guidelines, of the family history information. This is done via a patient questionnaire then verification against medical records and cancer registry. This study aims to quantify the benefits of this verification process.

Methods All consecutive GP referrals from Jan 2012 to end of Dec 2012 to the Nottingham Nurse led CFHSC were included in this study. Risk based on the family history data was assessed at three sources; based purely on GP information, based on patient questionnaire and finally following verification with the cancer registry and medical notes. Performance of GP referral and patient reported information was compared with the verified history using Chi Squared test.

Results 54 patients were included in the study. Using GP referral information alone categorised 12 high, 12 upper moderate, 30 lower moderate and no low risk of familial colorectal cancer. Using the patient reported family history screening questionnaire data resulted in 10 high, 11 upper moderate and 29 lower moderate and 4 low risk patients. Final verification with medical records resulted in 8 high, 9 upper moderate, 27 lower moderate and 10 low risk patients. Thus there was a significant reduction in number of colonoscopies required following verification of the family history with $10(18.5 \%$, Chi Squared 11.4, $\mathrm{p}<0.01)$ patients no longer requiring a colonoscopy based on their confirmed family history data compared with the GP history.

Conclusion The verification process adds significant value in reducing patient risk and preventing unnecessary screening procedures with an overall $18.5 \%$ reduction in colonoscopies requested compared to relying solely on the GP history.

Disclosure of Interest None Declared.

\section{PTH-006 EFFECT OF MEDICATION USE ON FAECAL OCCULT BLOOD TEST POSITIVITY IN THE NHS BOWEL CANCER SCREENING PROGRAMIME}

doi:10.1136/gutjnl-2013-304907.494

1,2,3,* M D Gill, ${ }^{2,3} \mathrm{M}$ Bramble, ${ }^{2,3,4} \mathrm{C}$ Rees. ${ }^{1}$ Northern Colorectal Cancer Audit Group, Northumberland; 'University of Durham, Durham; ${ }^{3}$ Northern Region Endoscopy Group, North East UK; ${ }^{4}$ South Tyneside Foundation Trust, South Tyneside, UK

Introduction Faecal occult blood test (FOBt) positivity is linked with both tumour site and gender. Left sided cancers, and cancers in men are detected in significantly greater proportions by screening. The aim of this study was to evaluate for an association with certain medication use at time of test, with the FOBt result, in patients diagnosed with a colorectal cancer.
Methods Using a regional colorectal cancer dataset (Northern Colorectal Cancer Audit Group) and Bowel Cancer Screening Programme database, all screen detected and interval cancers (diagnosed after a negative faecal occult blood test, before the next screening round) were identified. Diagnosis date was between April 2007 and March 2010. General Practitioners for each patient were asked to complete a proforma detailing use of hormone antagonists, hormone replacement therapy, anticoagulants, aspirin, nonsteroidal anti-inflammatory drugs (NSAIDs), and pre cancer diagnosis cholecystectomy. Medication use within two months of performing a FOBt was deemed positive. Chi-squared and logistic regression analyses were used.

Results Of 514 patients, 346 (67.3\%) proformas were returned and suitable for analysis. 120 patients analysed were in the interval cancer group, with 226 in the screen detected cancer group. Between screen detected and interval cancers groups, no difference was found in the use of hormone antagonists, hormone replacement therapy, anticoagulants, and aspirin. Rates of cholecystectomy were equivalent. The use of non-aspirin NSAIDs within two months of test was seen in a significantly greater proportion in the screen detected cancer group (10.6\% vs. $4.2 \%, p=0.039)$. For the population who used NA-NSAIDs, there was no difference between groups in gender, tumour location, or stage of tumour.

Conclusion The use of NA-NSAIDs around the time of test was associated with a greater rate of positivity of the FOBt. This finding adds to our understanding of factors influencing the positivity of FOB testing, and may be useful in understanding the rates of interval colorectal cancers within the screening programme.

Disclosure of Interest None Declared.

\section{PTH-007 TWO PHASE PHOTODYNAMIC ANTIMICROBIAL CHEMOTHERAPY (PACT) COMBINED WITH GERMINANT THERAPY EFFECTIVELY TREATS CLOSTRIDIUM DIFFICILE AND THEIR SPORES THAT ARE RESISTANT TO ANTIBIOTICS}

doi:10.1136/gutjnl-2013-304907.495

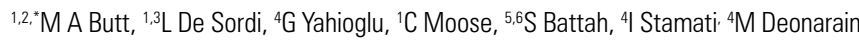
${ }^{3}$ E Allan. ${ }^{3 P}$ Mullany, ${ }^{1,2}$ L B Lovat. 'National Medical Laser Centre; ${ }^{2}$ Gastroenterology; ${ }^{3}$ Microbial Diseases, University College London; ${ }^{4}$ PhotoBiotics Ltd, Imperial College London, London; ${ }^{5}$ Organix Ltd; ${ }^{6}$ Department of Biological Sciences, University of Essex, Colchester, UK

Introduction Approximately $20 \%$ of patients with Clostridium diffcile (CD) infection relapse after initially effective treatment. These typically occur 3-10 days after cessation of standard antibiotic therapy with vancomycin or metronidazole. Some patients relapse 2 or even 3 times, each requiring additional courses of antibiotics. The general consensus is that relapse occurs either because $C D$ had not been completely eradicated by the antibiotics, or because spores are resistant to killing. Several days post-antibiotics, surviving spores transform into active bacterial forms again which multiply to produce toxins again. This study aimed to combine PACT, which we have shown to be effective against $C D$, with a novel strategy called germinant therapy.

Methods Germinant therapy with two phase PACT was evaluated against the hypervirulent R20291 strain of CD with photosensitisers (PS) we have found to effectively kill CD from earlier studies. Plates containing $\mathrm{CD}$ were treated with a single PACT treatment, pre and post germination of quiescent spores with the bile salt taurocholate. Results PACT effectively killed R20291 at doses $>10 \mu \mathrm{M}$ after exposure to laser light at $665 \mathrm{~nm}$ at an intensity of $24 \mathrm{~mJ} / \mathrm{cm} 2$. However, post PACT treatment of the C. difficile culture with the spore germinant taurocholate showed that $100 \%$ of CD spores were resistant to the treatment. Remarkably, it was shown that pre-incubation of CD spores in germination conditions for 30 minutes prior to PACT leads to $>99.9 \%$ kill of the initial number of spores permitting the killing of $\mathrm{CD}$ in both its vegetative and sporulating form. Moreover, toxicity of taurocholic acid was excluded in HT-29 colon cells. 